\title{
Entzündungen und Immunsystem
}

\section{Liebe Leserinnen, liebe Leser,}

die Bedeutung von Entzündungsprozessen als Ursache vieler chronischer Erkrankungen, v. a. vieler „Zivilisationskrankheiten" ist in den letzten Jahren immer deutlicher geworden. In zahlreichen internationalen Studien konnten chronische Entzündungsprozesse als Ursache oder wichtiger Teilaspekt von Arteriosklerose, Diabetes, Hypertonie, Morbus Parkinson, Alzheimer oder auch Krebserkrankungen aufgezeigt werden. Die für die Abwehr von Bakterien oder Viren sinnvollen komplexen Abwehr- und Entzündungsreaktionen werden auch durch viele andere Stressoren ausgelöst. Physische und psychische Stressfaktoren wie Schadstoffe (Tabakrauch!), Lärm, Strahlung (auch UV), Disstress (berufliche Überlastung) oder die ganz normale Nahrungsaufnahme und sportliche Betätigung induzieren z.B. die vermehrte Bildung von Sauerstoffradikalen und damit oxidativen Stress, die Aktivierung von Transferfaktoren (NF-kB) und die Freisetzung von Entzündungsmediatoren.

Die zum Schutz unseres Körpers vorgesehenen neuro-endokrino-immunologischen Abwehr- und Regulationsmechanismen, die den chronischen Entzündungsprozessen zugrunde liegen, geraten durch unseren Lebensstil (Stress, Erholungsdefizit, Genussgifte etc.) und unsere Lebensbedingungen (Schadstoffe, Strahlung, Lärm etc.) aus der Balance und führen zu Störungen und Schädigungen von wichtigen Stoffwechselprozessen mit daraus resultierenden Erkrankungen.
So wird die Arteriosklerose inzwischen nicht mehr als arterielles Fettablagerungsproblem, sondern als multifaktorielles, entzündlich-oxidatives Geschehen der Gewäßwand verstanden, bei der z.B. die Einlagerung von oxidiertem LDL nur ein Teilaspekt des komplexen Arteriosklerosegeschehens darstellt. Dazu passt auch die in den Studien der letzten Jahre gewonnene Erkenntnis, dass die viel gepriesenen Statine ihre Wirkung nicht über die Lipidsenkung, sondern durch antiinflammatorische Effekte erzielen.

Auch für neurodegenerative Erkrankungen wie Morbus Parkinson oder Morbus Alzheimer werden die ursächlichen Zusammenhänge mit inflammatorischen Prozessen immer deutlicher: Beim Morbus Parkinson addieren sich dabei die proinflammatorische Aktivität der im Bereich der dopaminergen Neurone stark vertretenen immunkompetenten, phagozytierenden Microglia mit der erhöhten oxidativen Belastung der dafür besonders vulnerablen Substantia nigra zu einem unheilvollen Cocktail. Schädigung und Untergang der dopaminergen Neurone sind die Folge.

Eine zentrale Bedeutung bei der Umsetzung interner (z.B. oxidativer Stress) und externer Stressoren (z.B. Mikroorganismen, Schadstoffe etc.) in körpereigene Abwehr- und Entzündungsreaktionen haben die Transferfaktoren, insbesondere NF-kB. Dies ist aus orthomolekularmedizinischer und präventiver Sicht sehr interessant, da die Aktivität von NF-kB (nukleäre Induktion der Synthese von Entzündungsmediatoren) durch verschiedene Nährstoffe gehemmt werden kann. Dazu zählen v.a. Glutathion, $\alpha$-Liponsäure, Omega-3Fettsäuren und Vitamin D. Dies sind genau jene Nährstoffe, bei denen wir eine weit verbreitete Mangelsituation sehen. Allein die Bedeutung eines ausreichenden Vitamin-D-Spiegels für die Prävention vieler „Zivilisationskrankheiten“ wird erst in den letzten Jahren erkannt. Vor dem Hintergrund eines epidemischen Vitamin-D-Mangels sind dies dramatische Erkenntnisse, die im klassisch-medizinischen Alltag sträflicherweise noch viel zu wenig berücksichtigt werden. Hier wird von politischer und medizinischer Seite schlicht fahrlässig gehandelt, da durch eine generelle Anhebung der Vitamin-D-Spiegel in der Bevölkerung viele der oben genannten „Zivilisationskrankheiten“ verhindert werden könnten. Umso mehr ist hier der Orthomolekularmediziner gefragt.

\section{Ihre Herausgeber}

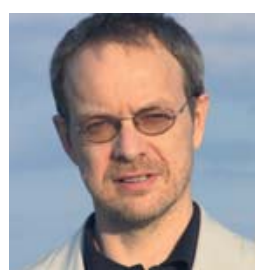

Dr. med. Hans-Peter Friedrichsen 